\title{
ECOLOGICAL INDICATOR VALUES OF SOME LICHEN SPECIES NOTED IN POLAND
}

\author{
JERZY FABISZEWSKI ${ }^{1}$, KATARZYNA SZCZEPAŃSKA ${ }^{2}$ \\ ${ }^{1}$ Department of Botany and Plant Ecology, \\ The Faculty of Life Sciences and Technology, \\ Wrocław University of Envirmental and Life Sciences \\ pl. Grunwaldzki 24A, 50-363 Wrocław, Poland \\ e-mail: jerzy.fabiszewski@up.wroc.pl \\ 2 The Main Library of Wrocław University of Environmental and Life Sciences \\ Norwida 29, 50-375 Wrocław, Poland
}

(Received: January 20, 2010. Accepted: October 7, 2010)

\begin{abstract}
The paper presents the ecological indicator values for 360 lichen species noted in Poland. For the given species estimated were the climatic indicators (light, temperature, moisture) and the edaphic ones (trophicity, habitat acidity). The estimate is based mainly on field studies and Polish lichenological literature. Presented are also the dynamics tendencies, i.e expansion or dieback of the analyzed species. According to papers by Polish authors, concerning various groups of plants, used were five degrees' scales of the analysed indices.
\end{abstract}

KEY WORDS: lichens, indicator values, Poland.

\section{INTRODUCTION}

From the beginning of scientific botanical studies it is generally known, that plants point at the habitat conditions in which they grow. That remark concerns also the fungi and other groups of living organisms. Astonishing is that before over half a century, under the influence of papers of the German geobotanicist H. Ellenberg and his disciples started were experiments of quantitative plant calibration on impact of various habitat factors. In 1965 (and succeeding years) elaborated were the so-called ecological indicator values for middle Europe plants (Ellenberg 1965). Since that time realized were many regional elaborations, mainly in country scale, among others also for Poland (Zarzycki et al. 2002). However, the lichens as a group have been elaborated by means of this method by V. Wirth not before the latest edition of Ellenberg's et al. (1992) monography. More recent observations and critical remarks concerning the use of ecological values for lichens are included, among others, in papers by Nimis et al. (2003, 2004). As opposed to plants the ecological lists of lichens are rarely published (both in local, as well as in country's scale). These lists allow better to understand the functioning of concentrating lichen ecosystems, as well as the scale of changes in the species composition of regional floras.

\section{MATERIAL AND METHOD}

The paper includes suggestions of ecological indicator values for 360 lichen species recorded in Poland (Table 1). The presented taxons are in most cases frequent or common and settle various types of natural forests and nonforests, both in lowland and in the mountains. For all the species evaluated were the climatic, light, temperature and moisture indicators, as well as the edaphic indicators: trophicity and habitat acidity. The indicator values have been elaborated on the basis of field studies and the accessible literature items describing more precise the habitat requirements or distribution of the particular taxons (e.g. Czarnota 2007; Ellenberg et. al. 1992; Fałtynowicz 2003; Nowak and Tobolewski 1975; Szczepańska 2008; Wirth 1995). Evatuated have been also the current dynamic tendencies observed in Poland (expansion or dieback of species), and for epiphytic lichens the tolerance of air contamination. We passed over the described by other authors response of lichens to heavy metals. The number of typical metalophytes in Poland is rather small. Furthermore, the views on metalophytic responses in lichens are considerably diverse (Ernst 2003). The critically endangered taxons, and those showing a drop in the sites has been determined on the basis of the "Red List of Extinct and endangered Lichens in Poland" (Ciesliński et al. 2003) The tax- 
TABLE 1. Ecological indicator values of some lichen species noted in Poland.

Species

Climate value Edaphic value Resistance Dynamic

Acarospora cervina A. Massal.

A. fuscata (Nyl.) Arnold

A. glaucocarpa (Ach.) Körb.

A. macrospora (Hepp) A. Massal. ex Bagl.

A. smaragdula (Wahlenb.) A. Massal.

Acrocordia gemmata (Ach.) A. Massal.

Alectoria nigricans (Ach.) Nyl.

A. ochroleuca (Hoffm.) A. Massal.

Allantoparmelia alpicola (Th. Fr.) Essl.

Allocetraria madreporiformis (Ach.) Kärnefelt \& A. Thell

Amandinea punctata (Hoffm.) Coppins \& Scheid.

Anaptychia ciliaris (L.) Körb. ex A. Massal.

Arctoparmelia incurva (Pers.) Hale

Arthonia radiata (Pers.) Ach.

A. spadicea Leight.

A. vinosa Leight.

Arthrorhaphis citrinella (Ach.) Poelt

Aspicilia caesiocinerea (Nyl. ex Malbr.) Arnold

A. calcarea (L.) Mudd

A. cinerea (L.) Körb.

A. contorta (Hoffm.)

A. laevata (Ach.) Arnold

A. moenium (Vain.) G. Thor \& Timdal

A. radiosa (Hoffm.) Poelt \& Leuckert

Bacidia arnoldiana Körb.

B. inundata (Fr.) Körb.

B. rosella (Pers.) De Not.

B. rubella (Hoffm.)A. Massal.

B. subincompta (Nyl.) Arnold

Baeomyces rufus (Huds.) Rebent.

Biatora amaurospoda Anzi

B. efflorescens (Hedl.) Räsänen

Bilimbia sabuletorum (Schreb.) Arnold

Brodoa atrofusca (Schaer.) Goward

B. intestiniformis (Vill.) Goward

Bryoria bicolor (Ehrh.) Brodo \& D. Hawksw.

B. fuscescens (Gyeln.) Brodo \& D. Hawksw.

Buellia aethalea (Ach.) Th. Fr.

B. disciformis (Fr.) Mudd

B. griseovirens (Turner \& Borrer ex Srn.) Almb.

Calicium glaucellum Ach.

C. salicinum Pers.

C. viride Pers

Caloplaca cerina (Hedw.) Th. Fr.

C. cirrochroa (Ach.) Th. Fr.

C. citrina (Hoffm.) Th. Fr.

C. decipens (Arnold) Blomb. \& Forssell

C. holocarpa (Hoffm.) A.E. Wade

C. saxicola (Hoffm.) Nordin

C. teicholyta (Ach.) J.Steiner

$\begin{array}{llllllll}\mathrm{L} & \mathrm{T} & \mathrm{W} & \mathrm{Tr} & \mathrm{R} & \mathrm{To} & \mathrm{E}\end{array}$

$\begin{array}{lllllll}5 & \mathrm{x} & 2 & 4 & 5 & - & 2\end{array}$

$\begin{array}{lllllll}5 & \mathrm{x} & 2 & 4 & 3 & - & 3\end{array}$

$\begin{array}{lllllll}4 & \mathrm{x} & 2 & 4 & 5 & - & 2\end{array}$

$\begin{array}{lllllll}5 & 3 & 1 & 3 & 5 & - & 2\end{array}$

$\begin{array}{lllllll}4 & \times & 3 & 4 & 4 & - & 3\end{array}$

$\begin{array}{lllllll}4 & 4 & 3 & 4 & 4 & 2 & 2\end{array}$

$\begin{array}{lllllll}5 & 1 & 4 & 1 & 1 & - & 2\end{array}$

$\begin{array}{lllllll}5 & 1 & 4 & 1 & 1 & - & 3\end{array}$

$\begin{array}{lllllll}5 & 1 & 4 & 1 & 1 & - & 2\end{array}$

$\begin{array}{lllllll}5 & 1 & 3 & 3 & 5 & - & 1\end{array}$

$\begin{array}{lllllll}4 & 4 & 2 & 4 & 3 & 5 & 5\end{array}$

$\begin{array}{lllllll}4 & 4 & 2 & 5 & 4 & 1 & 1\end{array}$

$\begin{array}{lllllll}5 & 2 & 3 & 1 & 2 & - & 3\end{array}$

$\begin{array}{lllllll}3 & 4 & 3 & 3 & 3 & 3 & 3\end{array}$

$\begin{array}{lllllll}2 & 4 & 4 & 3 & 2 & 4 & 3\end{array}$

$\begin{array}{lllllll}3 & 3 & 3 & 3 & 3 & 2 & 2\end{array}$

$\begin{array}{lllllll}5 & \mathrm{x} & 3 & 1 & 1 & - & 2\end{array}$

$\begin{array}{lllllll}5 & 3 & 3 & 4 & 3 & - & 3\end{array}$

$\begin{array}{lllllll}5 & 3 & 2 & 3 & 5 & - & 3\end{array}$

$\begin{array}{lllllll}5 & 3 & 3 & 3 & 3 & - & 3\end{array}$

$\begin{array}{llllllll}5 & 3 & 3 & 3 & 3 & - & 3\end{array}$

$\begin{array}{lllllll}2 & 3 & 5 & 2 & 2 & - & 3\end{array}$

$\begin{array}{lllllll}5 & 4 & 2 & 3 & 5 & - & 3\end{array}$

$\begin{array}{lllllll}5 & 4 & 1 & 5 & 5 & - & 3\end{array}$

$\begin{array}{lllllll}3 & 4 & 3 & 3 & 3 & 4 & 4\end{array}$

$\begin{array}{lllllll}2 & 3 & 5 & 3 & 4 & - & 3\end{array}$

$\begin{array}{lllllll}4 & 4 & 2 & 4 & 4 & 2 & 1\end{array}$

$\begin{array}{lllllll}4 & 4 & 3 & 4 & 4 & 2 & 2\end{array}$

$\begin{array}{lllllll}3 & 4 & 4 & 3 & 3 & 2 & 2\end{array}$

$\begin{array}{lllllll}3 & \mathrm{x} & 3 & 2 & 2 & - & 3\end{array}$

$\begin{array}{lllllll}4 & 2 & 3 & 1 & 1 & 3 & 3\end{array}$

$\begin{array}{lllllll}3 & 3 & 3 & 3 & 3 & 2 & 2\end{array}$

$\begin{array}{lllllll}3 & 3 & 3 & 4 & 5 & - & 3\end{array}$

$\begin{array}{lllllll}5 & 1 & 4 & 1 & 2 & - & 2\end{array}$

$\begin{array}{lllllll}5 & 1 & 4 & 1 & 2 & - & 2\end{array}$

$\begin{array}{lllllll}4 & 1-2 & 4 & 2 & 2 & 1 & 1\end{array}$

$\begin{array}{lllllll}4 & \mathrm{x} & 3 & 2 & 2 & 1 & 2\end{array}$

$\begin{array}{lllllll}5 & \mathrm{x} & 1 & 3 & 3 & - & 3\end{array}$

$\begin{array}{lllllll}3 & 3 & 3 & 3 & 3 & 2 & 2\end{array}$

$\begin{array}{lllllll}3 & 4 & 3 & 2 & 2 & 4 & 4\end{array}$

$\begin{array}{lllllll}2 & 3 & 3 & 2 & 2 & 2 & 2\end{array}$

$\begin{array}{lllllll}2 & 3 & 2 & 2 & 3 & 2 & 2\end{array}$

$\begin{array}{lllllll}2 & 3 & 2 & 2 & 2 & 2 & 2\end{array}$

$\begin{array}{lllllll}4 & 3 & 3 & 4 & 4 & 2 & 2\end{array}$

$\begin{array}{lllllll}5 & 1-3 & 2 & 5 & 5 & - & 2\end{array}$

$\begin{array}{lllllll}4 & 4 & 1 & 5 & 5 & - & 5\end{array}$

$\begin{array}{llllllll}5 & 4 & 1 & 5 & 5 & - & 4\end{array}$

$\begin{array}{lllllll}5 & 4 & 1 & 5 & 5 & - & 3\end{array}$

$\begin{array}{lllllll}5 & \mathrm{x} & 1 & 5 & 5 & - & 4\end{array}$

$\begin{array}{lllllll}5 & 4 & 1 & 5 & 5 & - & 4\end{array}$

$\begin{array}{lllllll}4 & 4 & 2 & 4 & 4 & 3 & 3\end{array}$ 
TABLE 1. Cont.

Species

Climate value Edaphic value Resistance Dynamic

Candelariella aurella (Hoffm.) Zahlbr.

C. reflexa (Nyl.) Lettau

C. vitellina (Hoffm.) Müll. Arg.

C. xanthostigma (Pers. ex Ach.) Lettau

Catillaria nigroclavata (Nyl.) Schuler

Catolechia wahlenbergii (Flot. ex Ach.) Körb.

Cetraria aculeata (Schreb.) Fr.

C. ericetorum Opiz

C. islandica (L.) Ach.

C. sepincola (Ehrh.) Ach.

Cetrelia olivetorum (Nyl.) W.L. Culb. \& C.F. Culb. s. lat.

Chaenotheca brachypoda (Ach.) Tibell

C. brunneola (Ach.) Müll. Arg.

C. chrysocephala (Turner ex Ach.) Th. Fr.

C. ferruginea (Turner ex Sm.) Mig.

C. furfuracea (L.) Tibell

C. phaeocephala (Turner) Th. Fr.

C. trichialis (Ach.) Th. Fr.

C. xyloxena Nádv.

Chrysothrix candelaris (L.) J.R. Laundon

C. chlorina (Ach.) J.R. Laundon

Cladonia arbuscula (Wallr.) Flot.

C. bellidiflora (Ach.) Schaer.

C. caespiticia (Pers.) Flörke

C. cenotea (Ach.) Schaer.

C. chlorophaea (Flörke ex Sommerf.) Spreng.

C. coccifera (L.) Willd.

C. coniocraea (Flörke) Spreng.

C. cornuta (L.) Hoffm.

C. deformis (L.) Hoffm.

C. digitata (L.) Hoffm.

C. fimbriata (L.) Fr.

C. foliacea (Huds.) Willd.

C. furcata (Huds.) Schrad.

C. gracilis (L.) Willd.

C. maci lenta Hoffm.

C. ochrochlora Flörke

C. phyllophora Hoffm.

C. pocillum (Ach.) Grognot

C. polydactyla (Flörke) Spreng.

C. portentosa (Dufour) Coem.

C. pyxidata (L.) Hoffm.

C. rangiferina (L.) F.H. Wigg.

C. stellaris (Opiz) Pouzar \& Vèzda

C. subulata (L.) F.H. Wigg.

C. sulphurina (Michx.) Fr.

C. uncialis (L.) F.H. Wigg.

Clauzadea monticola (Ach.) Hafellner \& Bellem.

Collema auriforme (With.) Coppins \& J.R. Laundon

C. crispum (Huds.) Weber ex F.H. Wigg.

C. cristatum (L.) Weber ex F.H. Wigg.

C. flaccidum (Ach.) Ach.

$\begin{array}{lllllll}\text { L } & \text { T } & \text { W } & \text { Tr } & \text { R } & \text { To } & \text { E }\end{array}$

$\begin{array}{lllllll}4 & 4 & 2 & 4 & 4 & 3 & 3\end{array}$

$\begin{array}{llllll}5 & \mathrm{x} & 2 & 3 & - & 3\end{array}$

$\begin{array}{lllllll}4 & 4 & 2 & 4 & 4 & 3 & 3\end{array}$

$\begin{array}{lllllll}4 & 4 & 2 & 4 & 4 & 3 & 3\end{array}$

$\begin{array}{lllllll}3 & 1 & 4 & 1 & 1 & - & 1\end{array}$


TABLE 1. Cont.

Species

Climate value Edaphic value Resistance Dynamic

C. limosum (Ach.) Ach.

C. tenax (Sw.) Ach.

Cornicularia normoerica (Gunn.) Du Rietz

Cyphelium tigillare (Ach.) Ach.

Cystocoleus ebeneus (Dillwyn) Thwaites

Dermatocarpon luridum (With:) J.R. Laundon

D. miniatum (L.) W. Mann

Dibaeis baeomyces (L. f.) Rambold \& Hertel

Dimelaena oreina (Ach.) Norm.

Dimerella pineti (Ach.) Vězda

Diploschistes muscorum (Scop.) R. Sant.

D. scruposus (Schreb.) Norman

Endocarpon pusillum Hedw.

Evernia divaricata (L.) Ach.

E. mesomorpha $\mathrm{Nyl}$.

E. prunastri (L.) Ach.

Fellhanera subtilis (Vèzda) Dieder. \& Sérus

Flavocetraria cucullata (Bellardi) Karnefelt \& A. Thell

F. nivalis (L.) Karnefelt \& Thell

Flavoparmelia caperata (L.) Hale

Flavopunctelia flaventior (Stirt.) Hale

Fulgensia fulgens (Sw.) Elenkin

Fuscidea austera (Nyl.) P. James

F. kochiana (Hepp) V. Wirth \& Vězda

F. pusilla Tönsberg

Graphis scripta (L.) Ach.

Gyalecta jenensis (Batseh) Zahlbr.

Hypocenomyce caradocensis (Leight. ex Nyl.) P. James \& Gotth. Schneid.

H. scalaris (Ach. ex Lilj.) M. Choisy

Hypogymnia farinacea Zopf

H. physodes (L.) Nyl.

H. tubulosa (Schaer.) Hav.

H. vittata (Ach.) Parrique

Hypotrachyna revoluta (Flörke) Hale

Icmadophila ericetorum (L.) Zahlbr.

Imshaugia aleurites (Ach.) S.L.F.Meyer

Lasallia pustulata (L.) Merat

Lecanactis abietina (Ach.) Körb.

Lecania cyrtella (Ach.) Th. Fr.

Lecanora albescens (Hoffm.) Branth \& Rostr.

L. argentata (Ach.) Malme

L. carpinea (L.) Vain.

L. cenisia Ach.

L. chlarotera $\mathrm{Nyl}$.

L. conizaeoides Nyl. ex Cromb.

L. crenulata (Dicks.) Hook.

L. dis persa (Pers.) Sommerf.

L. expallens Ach.

L. hagenii (Ach.) Ach.

L. intricata (Ach.) Ach.

L. intumescens (Rebent.) Rabenh.

\begin{tabular}{|c|c|c|c|c|c|c|}
\hline $\mathrm{L}$ & $\mathrm{T}$ & W & $\operatorname{Tr}$ & $\mathrm{R}$ & To & E \\
\hline 5 & 4 & 3 & 3 & 5 & - & 3 \\
\hline 4 & 4 & 4 & 3 & 5 & - & 3 \\
\hline 5 & 1 & 4 & 1 & 2 & - & 2 \\
\hline 5 & 2 & 3 & 1 & 1 & - & 1 \\
\hline 1 & 3 & 5 & 1 & 1 & - & 2 \\
\hline 4 & 3 & 5 & 2 & 3 & - & 1 \\
\hline 4 & 3 & 4 & 3 & 4 & - & 2 \\
\hline 5 & $\mathrm{x}$ & 1 & 1 & 1 & - & 2 \\
\hline 5 & 1 & 2 & 4 & 3 & - & 1 \\
\hline 1 & $\mathrm{x}$ & 5 & 2 & 2 & 4 & 4 \\
\hline 5 & $\mathrm{x}$ & 2 & 2 & 4 & - & 3 \\
\hline 3 & $\mathrm{x}$ & 3 & 3 & 2 & - & 3 \\
\hline 5 & $\mathrm{x}$ & 1 & 2 & 5 & - & 3 \\
\hline 3 & 2 & 4 & 1 & 2 & 1 & 1 \\
\hline 3 & 2 & 3 & 2 & 2 & 1 & 1 \\
\hline 4 & $\mathrm{x}$ & 3 & 4 & 3 & 2 & 2 \\
\hline 3 & $2-3$ & 4 & 2 & 2 & 3 & 3 \\
\hline 5 & 1 & 4 & 1 & 1 & - & 2 \\
\hline 5 & 1 & 4 & 1 & 1 & - & 1 \\
\hline 3 & 4 & 3 & 3 & 3 & 2 & 1 \\
\hline 4 & 3 & 2 & 4 & 3 & 1 & 1 \\
\hline 5 & 5 & 1 & 1 & 5 & - & 1 \\
\hline 5 & 2 & 3 & 1 & 1 & - & 3 \\
\hline 5 & 1 & 4 & 1 & 1 & - & 3 \\
\hline 3 & 3 & 3 & 3 & 3 & 3 & 3 \\
\hline 3 & 4 & 3 & 3 & 3 & 3 & 2 \\
\hline 2 & 3 & 4 & 3 & 5 & - & 2 \\
\hline 4 & 4 & 3 & 1 & 1 & 4 & 4 \\
\hline 4 & $\mathrm{x}$ & 2 & 1 & 1 & 5 & 5 \\
\hline 3 & 2 & 4 & 1 & 1 & 2 & 2 \\
\hline 4 & $\mathrm{x}$ & 3 & 2 & 2 & 5 & 4 \\
\hline 4 & 4 & 3 & 3 & 3 & 3 & 3 \\
\hline 3 & 2 & 4 & 1 & 1 & 1 & 1 \\
\hline 3 & 4 & 4 & 3 & 3 & 1 & 1 \\
\hline 3 & 2 & 4 & 1 & 1 & - & 2 \\
\hline 4 & 3 & 3 & 1 & 1 & 3 & 3 \\
\hline 5 & 4 & 1 & 4 & 3 & - & 1 \\
\hline 2 & 2 & 4 & 1 & 1 & 2 & 1 \\
\hline 4 & $\mathrm{x}$ & 2 & 4 & 4 & 3 & 3 \\
\hline 5 & 4 & 1 & 5 & 5 & - & 5 \\
\hline 4 & 4 & 3 & 3 & 3 & 3 & 3 \\
\hline 4 & 4 & 3 & 3 & 3 & 3 & 3 \\
\hline 5 & 2 & 4 & 1 & 1 & - & 3 \\
\hline 4 & 4 & 3 & 3 & 3 & 3 & 3 \\
\hline 3 & $\mathrm{x}$ & 2 & 2 & 1 & 5 & 5 \\
\hline 5 & 4 & 2 & 4 & 5 & - & 3 \\
\hline 5 & 4 & 1 & 5 & 5 & - & 5 \\
\hline 4 & 4 & 3 & 3 & 3 & 5 & 5 \\
\hline 4 & $\mathrm{x}$ & 2 & 4 & 4 & 4 & 3 \\
\hline 5 & $\mathrm{x}$ & 3 & 1 & 2 & - & 3 \\
\hline 4 & 4 & 3 & 3 & 3 & 2 & 2 \\
\hline 5 & 4 & 1 & 5 & 5 & - & 5 \\
\hline
\end{tabular}


TABLE 1. Cont.

\begin{tabular}{|c|c|c|c|c|c|c|c|}
\hline \multirow{2}{*}{ Species } & \multicolumn{3}{|c|}{ Climate value } & \multicolumn{2}{|c|}{ Edaphic value } & \multirow{2}{*}{$\frac{\text { Resistance }}{\text { To }}$} & \multirow{2}{*}{$\frac{\text { Dynamic }}{E}$} \\
\hline & $\mathrm{L}$ & $\mathrm{T}$ & $\mathrm{W}$ & $\operatorname{Tr}$ & $\mathrm{R}$ & & \\
\hline L. orosthea (Ach.) Ach. & 3 & 3 & 3 & 1 & 2 & - & 3 \\
\hline L. polytropa (Hoffm.) Rabenh. & 5 & $\mathrm{x}$ & 3 & 1 & 2 & - & 3 \\
\hline L. pulicaris (Pers.) Ach. & 3 & $\mathrm{x}$ & 3 & 3 & 2 & 4 & 4 \\
\hline L. rupicola (L.) Zahlbr. & 5 & 4 & 2 & 3 & 3 & - & 3 \\
\hline L. saligna (Schrad.) Zahlbr & 4 & $\mathrm{x}$ & 2 & 3 & 2 & 3 & 3 \\
\hline L. soralifera (Suza) Räsänen & 5 & 3 & 3 & 4 & 2 & - & 2 \\
\hline L. swartzii (Ach.) Ach. & 4 & 2 & 2 & 1 & 1 & - & 3 \\
\hline L. symmicta (Ach.) Ach. & 4 & $\mathrm{x}$ & 3 & 2 & 2 & 3 & 3 \\
\hline L. varia $(\mathrm{Hoffm}$.) Ach. & 4 & 4 & 2 & 3 & 3 & 3 & 3 \\
\hline Lecidea confluens (Weber) Ach. & 5 & 1 & 4 & 1 & 3 & - & 3 \\
\hline L. fuscoatra (L.) Ach. & 5 & 4 & 2 & 3 & 3 & - & 3 \\
\hline L. lactea Flörke ex Schaerer & 5 & 2 & 3 & 2 & 2 & - & 3 \\
\hline L. lapicida (Ach.) Ach. & 5 & 2 & 3 & 2 & 2 & - & 3 \\
\hline L. litophila (Ach.) Ach. & 4 & $\mathrm{x}$ & 3 & 1 & 2 & - & 3 \\
\hline L. plana (J. Lahm) Nyl. & 4 & $\mathrm{x}$ & 3 & 1 & 2 & - & 3 \\
\hline Lecidella elaeochroma (Ach.) M. Choisy & 3 & 4 & 3 & 3 & 3 & 3 & 3 \\
\hline L. stigmatea (Ach.) Hertel \& Leuckert & 5 & $\mathrm{x}$ & 2 & 4 & 5 & - & 4 \\
\hline Lecidoma demissum (Rutstr.) Gotth. Schneid. \& Hertel & 5 & 1 & 4 & 1 & 2 & - & 1 \\
\hline Lepraria caesioalba (B. de Lesd.) J.R. Laundon & 5 & 2 & 2 & 2 & 2 & - & 4 \\
\hline L. elobata Tönsberg & 3 & $\mathrm{x}$ & 3 & 2 & 2 & 5 & 4 \\
\hline L. incana (L.) Ach. & 3 & 4 & 3 & 2 & 2 & 5 & 5 \\
\hline L. jackii Tönsberg & 3 & 3 & 3 & 2 & 2 & 5 & 4 \\
\hline L. lobificans $\mathrm{Nyl}$. & 2 & $\mathrm{x}$ & 4 & 2 & 4 & 5 & 4 \\
\hline L. membranacea (Dicks.) Vain. & 2 & 3 & 3 & 2 & 2 & 5 & 4 \\
\hline L. neglecta (Nyl.) Lettau & 4 & $\mathrm{x}$ & 2 & 2 & 2 & - & 4 \\
\hline L. rigidula (B. de Lesd.) Tönsberg & 4 & 4 & 2 & 3 & 3 & 5 & 4 \\
\hline Leptogium gelatinosum (With.) J.R. Laundon & 3 & $\mathrm{x}$ & 4 & 3 & 5 & - & 2 \\
\hline L. lichenoides (L.) Zahlbr. & 3 & $\mathrm{x}$ & 4 & 3 & 4 & - & 3 \\
\hline L. saturninum (Dicks.) Nyl. & 4 & 3 & 3 & 3 & 4 & 1 & 1 \\
\hline Lichenomphalia hudsoniana (H.S. Jenn.) Redhead, Lutzoni, Moncalvo \& Vilgalys & 4 & 1 & 4 & 1 & 2 & - & 2 \\
\hline L. umbellifera (L.: Fr.) Redhead, Lutzoni, Moncalvo \& Vilgalys & 3 & 2 & 4 & 2 & 2 & - & 3 \\
\hline Lobaria pulmonaria (L.) Hoffm. & 3 & 3 & 4 & 3 & 3 & 1 & 1 \\
\hline Loxospora elatina (Ach.) A. Massal. & 3 & $2-3$ & 4 & 1 & 1 & 2 & 1 \\
\hline Melanelia hepatizon (Ach.) A. Thell & 5 & 1 & 4 & 1 & 1 & - & 3 \\
\hline M. stygia (L.) Essl. & 5 & 1 & 4 & 1 & 1 & - & 3 \\
\hline Melanelixia fuliginosa (Fr. ex Duby) O. Blanco, A. Crespo, Divakar, Essl., D. Hawksw. \& Lumbsch & 3 & 4 & 3 & 3 & 3 & 3 & 4 \\
\hline M. glabra (Schaer.) O. Blanco, A. Crespo, Divakar, Essl., D. Hawksw. \& Lumbsch & 4 & 3 & 2 & 5 & 4 & 1 & 1 \\
\hline M. subargentifera (Nyl.) O. Blanco, A. Crespo, Divakar, Essl., D. Hawksw. \& Lumbsch. & 4 & 4 & 2 & 5 & 4 & 2 & 2 \\
\hline Melanohalea exasperatula (Nyl.) O. Blanco, A. Crespo, Divakar, Essl., D. Hawksw. \& Lumbsch & 4 & 4 & 2 & 4 & 4 & 3 & 3 \\
\hline Menegazzia terebrata (Hoffm.) A. Massal. & 3 & 4 & 4 & 3 & 3 & 1 & 1 \\
\hline Micarea adnata Coppins & 2 & 2 & 4 & 2 & 2 & 2 & 2 \\
\hline M. botryoides (Nyl.) Coppins & 2 & 3 & 3 & 1 & 1 & 4 & 4 \\
\hline M. crassipes (Th. Fr.) Coppins & 5 & 1 & 4 & 1 & 1 & - & 2 \\
\hline M. denigrata (Fr.) Hedl. & 4 & $\mathrm{x}$ & 2 & 3 & 2 & - & 4 \\
\hline M. lignaria (Ach.) Hedl. & 4 & $1-2$ & 5 & 1 & 1 & - & 3 \\
\hline M. lithinella (Nyl.) Redl. & 2 & 3 & 4 & 1 & 1 & - & 3 \\
\hline M. lutulata (Nyl.) Coppins & 2 & 3 & 4 & 3 & 2 & - & 3 \\
\hline M. micrococca (Korb.) Gams ex Coppins & 2 & 4 & 4 & 2 & 1 & 4 & 3 \\
\hline M. peliocarpa (Anzi) Coppins \& R. Sant. & 2 & 3 & 4 & 2 & 1 & - & 4 \\
\hline M. prasina $\mathrm{Fr}$. & 2 & $\mathrm{x}$ & 4 & 1 & 1 & 5 & 5 \\
\hline M. sylvicola (Flot.) Vězda \& V. Wirth & 2 & 3 & 3 & 3 & 2 & - & 3 \\
\hline Mycobilimbia tetramera (De Not.) Vitik., Ahti, Kuusinen, Lommi \& T. Ulvinen & 3 & 4 & 3 & 3 & 3 & - & 3 \\
\hline
\end{tabular}


TABLE 1. Cont.

Species

Climate value Edaphic value Resistance Dynamic

Mycoblastus fucatus (Stirt.) Zahlbr.

\begin{tabular}{lllllll}
\hline $\mathrm{L}$ & $\mathrm{T}$ & $\mathrm{W}$ & $\mathrm{Tr}$ & $\mathrm{R}$ & $\mathrm{To}$ & $\mathrm{E}$
\end{tabular}

M. sanguinarius (L.) Norman

Nephroma parile (Ach.) Ach.

Normandina pulchella (Borrer) Nyl.

Ochrolechia androgyna (Hoffm.) Arnold

Opegrapha gyrocarpa Flotow

O. rufescens Pers.

O. varia Pers.

O. viridis (Ach.) Nyl.

O. vulgata (Ach.) Ach.

O. zonata Körb.

Ophioparma ventosa (L.) Norman

Pachyphiale fagicola (Hepp) Zwackh

Parmelia omphalodes (L.) Ach.

P. saxatilis (L.) Ach.

P. submontana Nadv. ex Hale

P. sulcata Taylor

Parmelina carporrhizans (Taylor) Poelt \& Vězda

P. tiliacea (Hoffm.) Hale

Parmeliopsis ambigua (Wulfen) Nyl.

P. hyperopta (Ach.) Arnold

Peltigera aphthosa (L.) Willd.

P. canina (L.) Willd.

P. degenii Gyeln.

P. didactyla (With.) J.R. Laundon

P. leucophlebia (Nyl.) Gyeln.

P. malacea (Ach.) Funck

P. praetextata (Flörke ex Sommerf.) Zopf

P. rufescens (Weiss) Humb.

P. venosa (L.) Hoffm.

Pertusaria albescens (Huds.) M. Choisy \& Werner

P. amara (Ach.) Nyl.

P. coccodes (Ach.) Nyl.

P. corallina (L.) Arnold

P. hemisphaerica (FIörke) Erichsen

P. hymenea (Ach.) Schaer.

P. lactea (L.) Arnold

P. leioplaca DC.

P. pertusa (Weigel) Tuck.

P. pupillaris (Nyl.) Th. Fr.

Phaeophyscia nigricans (Flörke) Moberg

P. orbicularis (Neck.) Moberg

Phlyctis argena (Spreng.) Flot.

Physcia adscendens H. Olivier

P. caesia (Hoffm.) Fürnr.

P. dubia (Hoffm.) Lettau

P. stellaris (L.) Nyl.

P. tenella (Scop.) DC.

Physconia distorta (With.) J.R. Laundon

P. enteroxantha (Nyl.) Poelt

P. grisea (Lam.) Poelt

P. muscigena (Ach.) Poelt

\begin{tabular}{|c|c|c|c|c|c|c|}
\hline $\mathrm{L}$ & $\mathrm{T}$ & W & $\mathrm{Tr}$ & 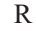 & & \\
\hline 3 & $\mathrm{x}$ & 3 & 2 & 1 & 5 & 5 \\
\hline 3 & 2 & 3 & 1 & 1 & 2 & 2 \\
\hline 3 & 3 & 4 & 3 & 3 & 1 & 1 \\
\hline 3 & 3 & 4 & 4 & 3 & 2 & 1 \\
\hline 3 & 3 & 3 & 3 & 3 & 2 & 2 \\
\hline 1 & 2 & 4 & 2 & 3 & - & 2 \\
\hline 2 & 4 & 3 & 3 & 3 & 2 & 2 \\
\hline 3 & 4 & 2 & 3 & 3 & 2 & 2 \\
\hline 2 & 4 & 4 & 3 & 3 & 2 & 2 \\
\hline 3 & 4 & 3 & 3 & 3 & 2 & 2 \\
\hline 1 & 3 & 4 & 2 & 3 & - & 2 \\
\hline 5 & 1 & 4 & 1 & 2 & - & 2 \\
\hline 4 & 4 & 2 & 4 & 3 & 3 & 2 \\
\hline 4 & 2 & 3 & 2 & 2 & - & 2 \\
\hline 4 & $\mathrm{x}$ & 3 & 2 & 2 & 3 & 3 \\
\hline 3 & 3 & 4 & 3 & 3 & 2 & 2 \\
\hline 4 & $\mathrm{x}$ & 3 & 3 & 3 & 4 & 3 \\
\hline 4 & 4 & 2 & 4 & 4 & 2 & 1 \\
\hline 4 & 4 & 2 & 4 & 4 & 2 & 2 \\
\hline 4 & $\mathrm{x}$ & 3 & 2 & 1 & 4 & 4 \\
\hline 4 & 2 & 3 & 1 & 1 & 2 & 2 \\
\hline 3 & 1 & 4 & 1 & 2 & - & 1 \\
\hline 3 & 4 & 3 & 3 & 4 & - & 2 \\
\hline 3 & 3 & 3 & 3 & 4 & - & 2 \\
\hline 5 & 4 & 2 & 4 & 4 & - & 4 \\
\hline 3 & 1 & 4 & 3 & 3 & - & 1 \\
\hline 4 & 3 & 4 & 1 & 2 & - & 2 \\
\hline 3 & 3 & 4 & 3 & 3 & - & 2 \\
\hline 5 & 4 & 2 & 3 & 5 & - & 4 \\
\hline 3 & 1 & 4 & 2 & 3 & - & 1 \\
\hline 4 & 4 & 2 & 4 & 4 & 3 & 3 \\
\hline 3 & 4 & 3 & 3 & 3 & 3 & 3 \\
\hline 4 & 4 & 3 & 4 & 3 & 2 & 2 \\
\hline 5 & $1-2$ & 3 & 2 & 2 & - & 3 \\
\hline 3 & 4 & 3 & 3 & 3 & 3 & 2 \\
\hline 4 & 4 & 3 & 3 & 3 & 2 & 1 \\
\hline 5 & $1-2$ & 3 & 2 & 2 & - & 3 \\
\hline 3 & 4 & 3 & 3 & 3 & 3 & 2 \\
\hline 3 & 4 & 3 & 3 & 3 & 2 & 2 \\
\hline 3 & 4 & 3 & 3 & 3 & 3 & 2 \\
\hline 5 & $\mathrm{x}$ & 1 & 5 & 5 & - & 4 \\
\hline 4 & $\mathrm{x}$ & 2 & 4 & 4 & 4 & 4 \\
\hline 4 & 4 & 2 & 3 & 3 & 4 & 3 \\
\hline 4 & $\mathrm{x}$ & 2 & 4 & 4 & 4 & 4 \\
\hline 5 & $\mathrm{x}$ & 1 & 5 & 5 & - & 4 \\
\hline 5 & $\mathrm{x}$ & 1 & 5 & 4 & - & 3 \\
\hline 4 & 4 & 3 & 4 & 4 & 2 & 2 \\
\hline 4 & $\mathrm{x}$ & 2 & 4 & 4 & 4 & 5 \\
\hline 4 & 4 & 2 & 5 & 4 & 1 & 1 \\
\hline 4 & 4 & 2 & 5 & 4 & 2 & 3 \\
\hline 4 & 4 & 2 & 4 & 4 & 4 & 4 \\
\hline 5 & 2 & 2 & 4 & 5 & - & 2 \\
\hline
\end{tabular}


TABLE 1. Cont.

Species

Climate value Edaphic value Resistance Dynamic

P. perisidiosa (Erichsen) Moberg

\begin{tabular}{lllllll}
\hline L & T & W & Tr & R & To & E
\end{tabular}

Placidium squamulosum (Ach.) Breuss

Placynthiella dasaea (Stirt.) Tønsberg

P. icmalea (Ach.) Coppins \& P. James

P. oligotropha (J.R. Laundon) Coppins \& P. James

P. uliginosa (Schrad.) Coppins \& P. James

Placynthium nigrum (Huds.) Gray

Platismatia glaca (L.) W.L. Culb. \& C.F. Culb.

Pleopsidium chlorophanum (Wahlenb.) Zopf

Pleurosticta acetabulum (Neck.) Elix \& Lumbsch

Polysporina simplex (Davies) Vězda

Porina aenea (Wallr.) Zahlbr.

P. chlorotica (Ach.) Mull. Arg.

P. leptalea (Durieu \& Mont.) A.L. Srn.

Porpidia crustulata (Ach.) Hertel \& Knoph

P. macrocarpa (DC.) Hertel \& A.J. Schwab

P. tuberculosa (Srn.) Hertel \& Knoph

Protoblastenia rupestris (Scop.) J. Steiner

Protopannaria pezizoides (Weber) P. M. Jørg. \& S. Ekman

Protoparmelia badia (Hoffm.) Hafellner

Pseudephebe pubescens (L.) M. Choisy

Pseudevernia furfuracea (L.) Zopf

Psilolechia lucida (Ach.) M. Choisy

Psora decipiens (Hedw.) Hoffm.

P. testacea Hoffm.

Punctelia jeckeri (Roum.) Kalb

P. subrudecta (Nyl.) Krog

Pycnothelia papillaria Dufour

Pyrenula nitida (Weigel) Ach.

P. nitidella (Schaer.) Müll. Arg.

Ramalina capitata (Ach.) Nyl.

R. carpatica Körb.

R. farinacea (L.) Ach.

R. fastigiata (Pers.) Ach.

$R$. fraxinea $(\mathrm{L}$.) Ach.

R. pollinaria (Westr.) Ach.

Rhizocarpon alpicola (Anzi) Rabenh.

$R$. distinctum Th. Fr.

R. geographicum (L.) DC.

$R$. hochstetteri (Körb.) Vain.

R. lecanorinum Anders

R. polycarpum (Hepp) Th. Fr.

Rhizoplaca chrysoleuca (Sm.) Zopf

Rinodina pyrina (Ach.) Arnold

Ropalospora viridis (Tönsberg) Tönsberg

Sarcogyne privigna (Ach.) A. Massal.

S. reguralis Körb.

Schaereria fuscocinerea (Nyl.) Clauzade \& Cl. Roux

Scoliciosporum chlorococcum (Graewe ex Stenh.) Vĕzda

S. umbrinum (Ach.) Arnold

Solorina crocea (L.) Ach.

S. saccata (L.) Ach.

\begin{tabular}{|c|c|c|c|c|c|c|}
\hline 4 & 4 & 2 & 5 & 4 & 2 & 1 \\
\hline 5 & 4 & 1 & 4 & 5 & - & 2 \\
\hline 3 & $x$ & $\mathrm{x}$ & 1 & 1 & - & 5 \\
\hline 4 & $x$ & $\mathrm{x}$ & 1 & 1 & - & 5 \\
\hline 5 & $\mathrm{x}$ & 1 & 1 & 1 & - & 4 \\
\hline 5 & $\mathrm{x}$ & 1 & 1 & 1 & - & 4 \\
\hline 5 & $\mathrm{x}$ & 2 & 3 & 5 & - & 2 \\
\hline 4 & $\mathrm{x}$ & 3 & 2 & 2 & 3 & 3 \\
\hline 4 & $1-2$ & 2 & 1 & 1 & - & 1 \\
\hline 4 & 4 & 2 & 5 & 4 & 1 & 1 \\
\hline 5 & 4 & 2 & 3 & 3 & - & 3 \\
\hline 2 & 4 & 4 & 3 & 3 & 4 & 4 \\
\hline 2 & 3 & 5 & 2 & 2 & - & 3 \\
\hline 2 & 3 & 4 & 3 & 3 & 3 & 2 \\
\hline 4 & $x$ & 2 & 1 & 2 & - & 3 \\
\hline 4 & $2-3$ & 3 & 1 & 2 & - & 3 \\
\hline $\mathrm{x}$ & $\mathrm{x}$ & 3 & 1 & 2 & - & 3 \\
\hline 4 & $\mathrm{x}$ & 3 & 4 & 5 & - & 3 \\
\hline 2 & 2 & 5 & 2 & 2 & - & 1 \\
\hline 5 & $1-2$ & 3 & 2 & 2 & - & 2 \\
\hline 5 & 1 & 4 & 1 & 1 & - & 1 \\
\hline 4 & $\mathrm{x}$ & 3 & 2 & 1 & 3 & 3 \\
\hline 1 & 3 & 2 & 2 & 2 & - & 3 \\
\hline 5 & $\mathrm{x}$ & 1 & 2 & 5 & - & 1 \\
\hline 5 & $\mathrm{x}$ & 1 & 2 & 5 & - & 1 \\
\hline 4 & 4 & 2 & 4 & 4 & 3 & 2 \\
\hline 4 & 4 & 2 & 4 & 4 & 3 & 2 \\
\hline 5 & $\mathrm{x}$ & 1 & 1 & 1 & - & 1 \\
\hline 3 & 4 & 3 & 3 & 3 & 2 & 2 \\
\hline 3 & 4 & 3 & 3 & 3 & 2 & 1 \\
\hline 5 & $\mathrm{x}$ & 3 & 4 & 3 & - & 1 \\
\hline 5 & 1 & 4 & 1 & 1 & - & 2 \\
\hline $3-4$ & 4 & 2 & 4 & 4 & 2 & 2 \\
\hline 4 & 4 & 2 & 4 & 4 & 2 & 2 \\
\hline 4 & 4 & 2 & 4 & 4 & 2 & 2 \\
\hline 4 & 4 & 2 & 4 & 4 & 2 & 2 \\
\hline 5 & 1 & 4 & 1 & 2 & - & 2 \\
\hline 5 & 4 & 2 & 3 & 3 & - & 3 \\
\hline 5 & $\mathrm{x}$ & 3 & 2 & 1 & - & 3 \\
\hline 4 & 1 & 4 & 1 & 2 & - & 2 \\
\hline 5 & $\mathrm{x}$ & 2 & 2 & 2 & - & 3 \\
\hline 4 & $\mathrm{x}$ & 3 & 2 & 3 & - & 3 \\
\hline 5 & 1 & 4 & 4 & 3 & - & 1 \\
\hline 4 & $\mathrm{x}$ & 3 & 4 & 4 & 3 & 3 \\
\hline 3 & 4 & 4 & 3 & 2 & 4 & 4 \\
\hline 4 & 3 & 2 & 4 & 4 & - & 2 \\
\hline 5 & 4 & 1 & 3 & 5 & - & 4 \\
\hline 5 & $1-2$ & 3 & 1 & 2 & - & 3 \\
\hline 3 & $\mathrm{x}$ & 3 & 2 & 1 & 5 & 5 \\
\hline 4 & $\mathrm{x}$ & 3 & 3 & 3 & - & 4 \\
\hline 5 & 1 & 4 & 1 & 1 & - & 1 \\
\hline 3 & $\mathrm{x}$ & 5 & 5 & 5 & - & 2 \\
\hline
\end{tabular}


TABLE 1. Cont.

Species

Climate value Edaphic value Resistance Dynamic

Sphaerophorus fragilis (L.) Pers.

Staurothele frustulenta Vain.

Steinia geophana (Nyl.) Stein

Stereocaulon dactylophyllum Flörke

S. nanodes Tuck.

S. vesuvianum Pers.

Strangospora moriformis (Ach.) Stein

S. pinicola (A. Massal.) Körb.

Tephromela atra (Huds.) Hafellner

Thamnolia vermicularis (Sw.) Schaerer

Thelocarpon laureri (Flot.) Nyl.

Thelomma ocellatum (Körber) Tibell

Thelotrema lepadinum (Ach.) Ach.

Thrombium epigaeum (Pers.) Wallr.

Toninia candida (Weber) Th. Fr.

T. sedifolia (Scop.) Timdal

Trapelia coarctata (Turner ex Sm.) M. Choisy

T. glebulosa (Srn.) J.R. Laundon

T. obtegens (Th. Fr.) Hertel

T. placodioides Coppins \& P. James

Trapeliopsis flexuosa (Fr.) Coppins \& P. James

T. gelatinosa (Flörke) Coppins \& P. James

T. granulosa (Hoffm.) Lumbsch

T. pseudogranulosa Coppin s \& P. James

Tremolecia atrata (Ach.) Hertel

Tuckermanopsis chlorophylla (Willd.) Hale

Umbilicaria crustulosa (Ach.) Frey

U. cylindrica (L.) Delise ex Duby

U. deusta (L.) Baumg.

U. hirsuta (Sw. ex Westr.) Hoffm.

U. hyperborea (Ach.) Hoffm.

U. polyphylla (L.) Baumg.

Usnea filipendula Stirt.

$\begin{array}{ccccccc}\mathrm{L} & \mathrm{T} & \mathrm{W} & \mathrm{Tr} & \mathrm{R} & \text { To } & \mathrm{E} \\ 4 & 2 & 4 & 4 & 5 & & \end{array}$

U. hirta (L.) Weber ex F.H. Wigg.

U. subfloridana Stirt.

Verrucaria muralis Ach.

V. nigrescens Pers.

Vulpicida pinastri (Scop.) J.-E. Mattsson \& M.J. Lai

V. tubulosus (Schaer.) J.-E. Mattsson \& M.J. Lai

Xanthoparmelia conspersa (Ehrh. ex Ach.) Hale

$X$. loxodes (Nyl.) O. Blanco et al.

X. stenophylla (Ach.) Ahti \& D. Hawksw.

Xanthoria candelaria (L.) Th. Fr.

X. elegans (Link) Th. Fr.

$X$. fallax (Hepp) Arnold

$X$. parietina $(\mathrm{L}$.) Th. Fr.

X. polycarpa (Hoffm.) Th. Fr. ex Rieber

\begin{tabular}{|c|c|c|c|c|c|c|}
\hline 4 & 2 & 4 & 4 & 5 & - & 1 \\
\hline 5 & 1 & 4 & 2 & 2 & - & 1 \\
\hline 5 & 4 & 1 & 5 & 4 & - & 3 \\
\hline 5 & 4 & 3 & 3 & 3 & - & 3 \\
\hline 5 & $1-2$ & 3 & 2 & 2 & - & 1 \\
\hline 5 & 3 & 2 & 3 & 2 & - & 1 \\
\hline 5 & $1-2$ & 3 & 2 & 3 & - & 2 \\
\hline 4 & 4 & 2 & 3 & 3 & 3 & 3 \\
\hline 4 & 4 & 2 & 3 & 3 & 3 & 3 \\
\hline 5 & $\mathrm{x}$ & 3 & 3 & 3 & - & 2 \\
\hline 5 & 1 & 4 & 1 & 1 & - & 3 \\
\hline 4 & $\mathrm{x}$ & 3 & 4 & 4 & - & 3 \\
\hline 5 & 4 & 3 & 3 & 3 & - & 3 \\
\hline 3 & $2-3$ & 3 & 3 & 3 & 2 & 2 \\
\hline 4 & 3 & 2 & 3 & 4 & - & 3 \\
\hline 5 & $\mathrm{x}$ & 1 & 2 & 5 & - & 1 \\
\hline 5 & $\mathrm{x}$ & 1 & 2 & 5 & - & 2 \\
\hline 3 & 4 & 3 & 2 & 2 & - & 3 \\
\hline 3 & 4 & 3 & 2 & 2 & - & 3 \\
\hline 4 & 4 & 2 & 2 & 3 & - & 3 \\
\hline 3 & 4 & 3 & 2 & 3 & - & 3 \\
\hline 4 & $\mathrm{x}$ & $x$ & 1 & 1 & - & 4 \\
\hline 2 & 3 & 4 & 1 & 1 & - & 3 \\
\hline 5 & $\mathrm{x}$ & 2 & 1 & 1 & - & 4 \\
\hline 3 & 4 & 3 & 1 & 1 & - & 3 \\
\hline 5 & $1-2$ & 3 & 2 & 1 & - & 3 \\
\hline 4 & $\mathrm{x}$ & 3 & 2 & 2 & 2 & 2 \\
\hline 5 & 1 & 4 & 2 & 2 & - & 2 \\
\hline 5 & $1-2$ & 3 & 2 & 2 & - & 3 \\
\hline 5 & $\mathrm{x}$ & 3 & 3 & 3 & - & 3 \\
\hline 5 & $2-3$ & 2 & 3 & 3 & - & 3 \\
\hline 5 & $1-2$ & 3 & 2 & 2 & - & 2 \\
\hline 5 & $\mathrm{x}$ & 3 & 3 & 2 & - & 3 \\
\hline 3 & $3-4$ & 4 & 2 & 2 & 1 & 2 \\
\hline 4 & 4 & 4 & 2 & 2 & 1 & 2 \\
\hline 3 & $3-4$ & 3 & 3 & 3 & 1 & 2 \\
\hline 5 & $\mathrm{x}$ & 2 & 4 & 5 & - & 3 \\
\hline 5 & $\mathrm{x}$ & 2 & 4 & 5 & - & 4 \\
\hline 4 & $\mathrm{x}$ & 3 & 1 & 1 & 2 & 2 \\
\hline 5 & 1 & 3 & 3 & 5 & - & 1 \\
\hline 5 & 4 & 2 & 4 & 3 & - & 3 \\
\hline 5 & 4 & 2 & 4 & 3 & - & 3 \\
\hline 5 & 4 & 2 & 4 & 3 & - & 3 \\
\hline 4 & 4 & 2 & 4 & 4 & 3 & 3 \\
\hline 5 & 3 & 1 & 5 & 5 & - & 3 \\
\hline 4 & 4 & 2 & 4 & 4 & 3 & 2 \\
\hline 4 & 4 & 2 & 4 & 4 & 4 & 3 \\
\hline 4 & 4 & 2 & 4 & 4 & 4 & 3 \\
\hline
\end{tabular}

onomy of lichens has been accepted after Smith et al. (2009), and for certain taxons after Diederich et al. (2010) and Fałtynowicz (2003).
The indicators describe the site requirements in every of the species, the most typical conditions in which the species is most frequent found and develops best. All the 
presented indicators have five-degree scales, and in the authors' intention they ought to refer to the paper of Zarzycki et al. (2002), and facilitate the possible statistical breakdowns and conversions. The scales with more than five degrees are, in our opinion, at the present stage of knowledge of lichen ecology are difficult for evaluation. The indicators do not reflect the whole range of site conditions, in which the given taxon can be met, and can in great extent be recognized as subjective perceptions of the authors, based on their individual experience acquired in field studies. However, one should remember that the presented indicators are only a certain proposal and an encouragement and invitation for a broader scientific discussion.

\section{EXPLANATIONS \\ OF COLUMN HEADINGS AND SYMBOLS}
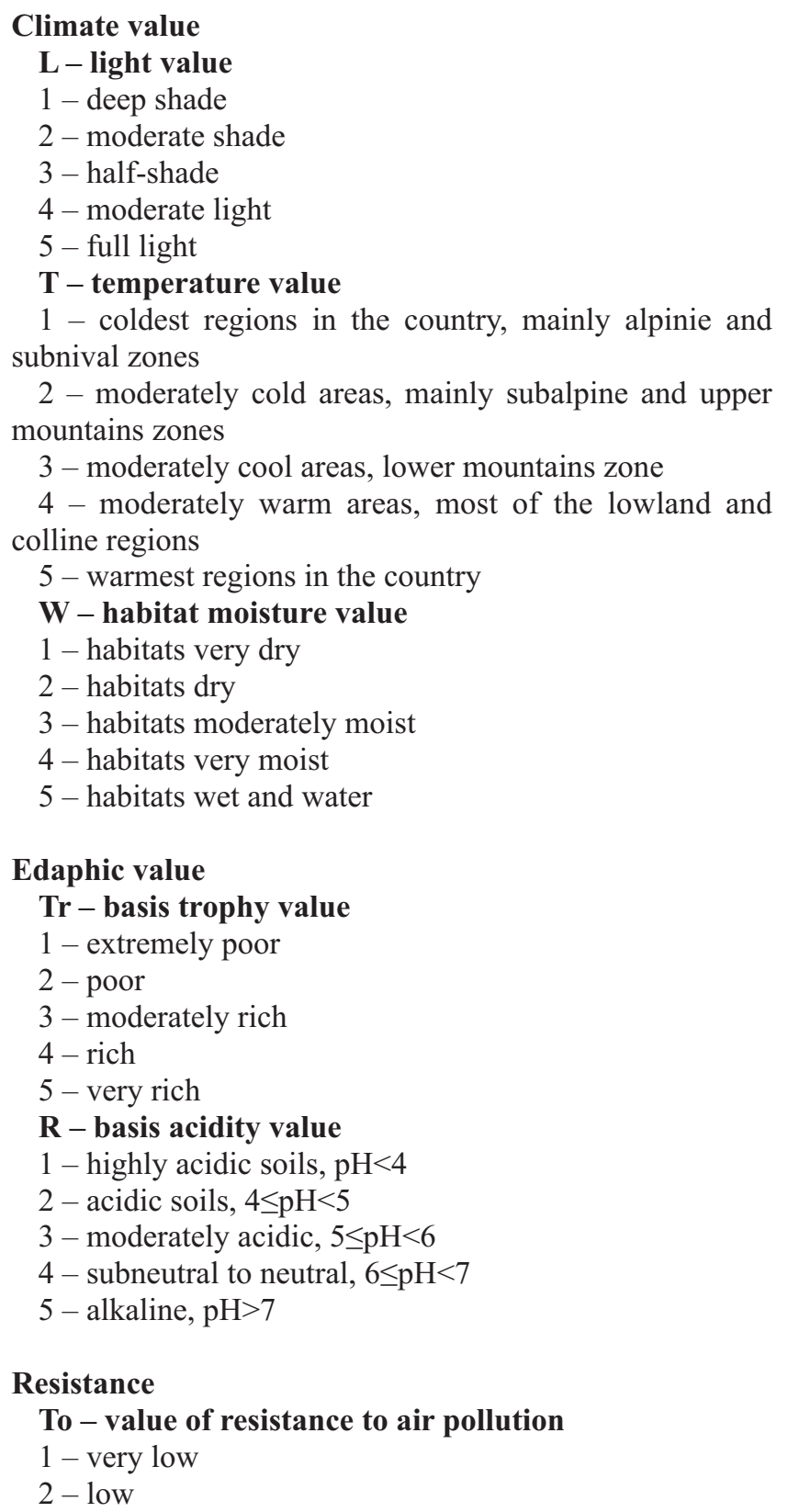

$$
\begin{aligned}
& 3 \text { - moderately } \\
& 4 \text { - high } \\
& 5 \text { - very high }
\end{aligned}
$$

\section{Dynamic}

\section{$\mathbf{E}$ - dynamic tendencies in the last decade}

1 - marked decrease in the number of localities, endangered species

2 - decrease in the number of localities

3 - no marked change in the number of localities observable, disappearance and appearance of new localities

4 - increase in the number of localities

5 - considerable increase and occupation of new localities, strongly expanding species

$$
\begin{aligned}
& -- \text { not investigated } \\
& \mathrm{x}-\text { very varied value }
\end{aligned}
$$

\section{LITERATURE CITED}

CIEŚLIŃSKI S., CZYŻEWSKA K., FABISZEWSKI J. 2003. Czerwona lista porostów wymarłych i zagrożonych w Polsce. In: Czyżewska K. (ed.), Zagrożenie porostów w Polsce. Monogr. Bot. 91: 14-49.

CZARNOTA P. 2007. The lichen genus Micarea (Lecanorales, Ascomycota) in Poland. Pol. Bot. Stud. 23: 1-199.

DIEDERICH P., ERTZ D., STAPPER N., SÉRUSIAUX E., BROECK D. VAN DEN, BOOM P. VAN DEN, RIES C. 2010. The lichens and lichenicolous fungi of Belgium, Luxembourg and northern France. URL: http://www.lichenology.info

ELLENBERG H. 1965. Zeigerpflanzen im Landwirtschaftsbereich. Ber. Geob. Inst. ETH, Stiftung Rübel 36: 121-176.

ELLENBERG H., WEBER H.E., DÜLL R., WIRTH V., WERNER W., PAULISSEN D. 1992. Zeigerwerte von Pflanzen in Mitteleuropa. Scripta Geobot. 18: 1-248.

ERNST W.H.O. 2003. The use of higher plants as bioindicators. In: Market B.A., Breure A.M. (eds), Bioindicators and Biomonitors, p. 423-463. Elsevier, Amsterdam.

FAŁTYNOWICZ W. 2003. Lichens, lichenicolous and allied fungi of Poland. An annotated checklist. W. Szafer Institute of Botany, Polish Academy of Sciences, Kraków, pp. 435.

NIMIS P.L., SCHEIDEGGER C., WOLSELEY P.A. (eds). 2002. Monitoring with lichens - Monitoring lichens. NATO Sci. Series 7. Kluwer Acad. Publ., Dordrecht, pp. 408.

NIMIS P.L., MARTELLOS S. 2004. Keys to the lichens of Italy. I. Terricolous species. Edizioni Goliardiche, Trieste, pp. 341.

NOWAK J., TOBOLEWSKI Z. 1975. Porosty polskie. PWN, Warszawa-Kraków, pp. 1173.

SMITH C.W., APTROOT A., COPPINS B.J., FLETCHER A., GILBERT O.L., JAMES P.W., WOLSELEY P.A. 2009. The lichen of Great Britain and Ireland. British Lichen Society, London, pp. 1046.

SZCZEPAŃSKA K. 2008. Antropogeniczne przemiany bioty porostów Masywu Śnieżnika i Gór Bialskich. Acta Bot. Siles. Monogr. 4: 1-291.

WIRTH V. 1995. Die Flechten Baden-Württembergs. II Aufl. Stuttgart, Verl. Eugen Ulmer, Stuttgart, pp. 1006.

ZARZYCKI K., TRZCIŃSKA-TACIK H., RÓŻAŃSKI W., SZELĄG Z., WOŁEK J., KORZENIAK U. 2002. Ecological indicator values of vascular plants of Poland. W. Szafer Institute of Botany, Polish Academy of Sciences, Kraków, pp. 183. 